\title{
Outcomes of Surgical Treatment of Skin Cancer at Surgical Oncology Unit of Donka, Conakry University Hospital
}

\author{
Bangaly Traore ${ }^{1 *}$, Leopold Lamah ${ }^{2}$ \\ ${ }^{1}$ Surgical Oncology Unit, National Hospital of Donka, Conakry University Hospital, Conakry, Guinea \\ ${ }^{2}$ Department of Orthopedics and Traumatology, National Hospital of Donka, Conakry University Hospital, Conakry, Guinea \\ Email: *ucodonka@gmail.com
}

How to cite this paper: Traore, B. and Lamah, L. (2017) Outcomes of Surgical Treatment of Skin Cancer at Surgical Oncology Unit of Donka, Conakry University Hospital. Journal of Cancer Therapy, 8, 1086-1094.

https://doi.org/10.4236/jct.2017.812093

Received: October 11, 2017

Accepted: December 1, 2017

Published: December 4, 2017

Copyright (๑) 2017 by authors and Scientific Research Publishing Inc. This work is licensed under the Creative Commons Attribution International License (CC BY 4.0).

http://creativecommons.org/licenses/by/4.0/

(c) (i) Open Access

\begin{abstract}
Aim: The main aim of this study was to evaluate outcomes after surgical treatment of skin cancer. Methods: This retrospective cohort study concerned 46 patients who presented 43 skin cancers lesions surgically treated at surgical oncology unit, Conakry University Hospital. There were 29 (61.7\%) squamous cell carcinoma, 12 (25.5\%) melanoma, 4 (8.5\%) sarcomas and 1 (2.1\%) porocarcinoma. Surgical indications and prognosis factors were analyzed. Results: Surgical treatment included simple excision in 2 cases $(3.8 \%)$, wide excision in 38 cases $(71.7 \%)$ and amputation/disarticulation in 13 cases $(24.5 \%)$. Inguinal lymph node dissection was performed in 16 patients $(34.3 \%)$. Postoperative complications were wound suppuration (5 cases), lower limb lymphedema (4 cases), seroma (2 cases) and skin flap necrosis (1 case). Surgical margins were free in $28(60.9 \%)$ patients, infiltrated in 3 patients $(6.5 \%)$ and unspecified in 15 patients (32.6\%). The median follow-up after surgery was 29 months. During the follow-up, 13 patients $(28.3 \%)$ had a relapse. The relapse was influenced by surgical margins $(p=0.012)$ and iterative resection $(p=0.04)$. Overall survival was $65.2 \%$. Factors related to survival in univariate analysis were: iterative resection $(p=0.008)$, fungated tumor $(p=0.037)$, the status of surgical margins $(p=0.002)$ and the occurrence of relapse $(p=0.0000)$. In multivariate analysis, the status of surgical margins was the only independent prognostic factor. Conclusion: The prognosis after surgical treatment of cutaneous cancers depends on the resection margins.
\end{abstract}

\section{Keywords}

Skin Cancer, Surgery, Prognosis 


\section{Background}

Skin cancer is one of the common cancers in our setting. It accounted $7.8 \%$ of all cancer registered at surgical oncology unit of Donka, Conakry University Hospital [1]. Regardless of the type of skin cancer, the age-related incidence is increasing worldwide [2] [3]. They are histological varieties with different clinical and prognostic expressions. The high prevalence of squamous cell carcinomas and melanoma in blacks unlike basal cell carcinomas among whites was reported by several studies [4] [5] [6]. In our context, skin cancers are of late diagnosis. Initially, some were often taken care of prior to consultation in the specialized settings, which complicates the evolution in advanced stage [1] [7]. Surgery is the most accessible means of treatment. Surgical removal is the standard treatment for non-metastatic skin cancer. The surgical modalities depend on the primary site, the segment of the affected limb, the invasion of adjacent tissues (nodes, muscles, and bones), the general condition of patients and the patients wish. This surgical treatment could be supplemented in some cases by radiotherapy [7] which is not available in our country. This study aimed to evaluate outcomes factors after surgical treatment of skin cancer in our unit.

\section{Material and Methods}

\section{Study Design}

We carried out a retrospective cohort study of cutaneous cancers operated from 2007 to 2014 at the surgical oncology unit of Donka, Conakry university hospital.

We recorded skin cancers histologically confirmed and then described the clinicopathological characteristics, indications and surgical treatment outcomes.

- Inclusion criteria

We included all skin cancers histological confirmed and operated during study period while non-operated skin cancers have been excluded.

- Data collection

Sociodemographic data were age, sex and albinism. An iterative excision, tumor fungating, tumor size, primary site, regional lymph node and clinical stage (according TNM UICC 2010) [8] were analyzed as clinicopathological characteristics.

For skin cancer surgery, we collected surgical removal modalities, lymph node dissection, surgical margins, wound closure, surgical complications and hospital staying. Lymph node dissection was performed in cases of regional lymph node involvement. The follow up period was assessed from the date of surgery to the latest news. Adjuvant chemotherapy was prescribed for patients with surgical margin involvement or in case of high risk of recurrence. The parameters evaluated during this follow up were surgical complications, relapse, survival, and prognosis factors.

\section{Study Analysis}

Category variables were shown as the frequency (\%), and continuous variables were presented as the median and interquartile range (IQR). Survival was stu- 
died according to the Kaplan-Meier method. The factors of recurrence and survival were evaluated by univariate analysis using the Fisher exact test and Log Rank test. The independent prognostic factors were assessed by multivariate analysis using the Cox model. The difference was significant if the $p$ value was less than 0.05. Statistic data analysis was done using SPSS software version 17.0 (SPSS, Inc, Chicago, IL).

\section{Ethical Considerations}

In this retrospective study, data were collected anonymously and confidentially.

\section{Results}

From 2007 to 2014, 102 histologically confirmed skin cancers were recorded in the surgical Oncology Unit of Donka, teaching hospital Conakry. In this retrospective cohort study, data of 46 patients operated during this period were analyzed. A total of 46 patients were included. There were 29 cases (61.7\%) of squamous cell carcinoma, 12 cases $(25.5 \%)$ of melanoma, 4 cases $(8.5 \%)$ of sarcomas, and 1 case $(2.1 \%)$ of porocarcinoma. Table 1 and Table 2 present respectively the clinicopathological characteristics and the surgical treatment methods carried out for these 4 types of skin cancers.

\section{Squamous cell carcinoma}

There were 36 squamous cell carcinoma lesions in 29 patients. Most common primary site was head and neck 17 (58.6\%) and lower limbs 13 (44.8\%). There

Table 1. Clinicopathological characteristics of skin cancer operated.

\begin{tabular}{ccccc}
\hline & $\begin{array}{c}\text { SCC } \\
\mathrm{n}=29 \mathrm{pts}\end{array}$ & $\begin{array}{c}\text { Melanoma } \\
\mathrm{n}=12\end{array}$ & $\begin{array}{c}\text { Sarcoma } \\
\mathrm{n}=4\end{array}$ & $\begin{array}{c}\text { Porocacinoma } \\
\mathrm{n}=1\end{array}$ \\
\hline Median age & 48.0 & 71.0 & 47.5 & $1: 3$ \\
Male to female ratio & $1: 1.4$ & $1: 2$ & $1(25.0 \%)$ & \\
Albinos & $10(34.5 \%)$ & 0 & 4 \\
Number of lesions & 36 & 12 & $2(50.0)$ \\
Primary site & & $1(8.3)$ & 0 \\
Head and neck & $10(34.5)$ & $1(8.3)$ & 0 \\
Upper limb & $4(13.8)$ & $10(83.4)$ & $2(50.0)$ \\
Trunck & $2(7.0)$ & $9(75.0)$ & $4(100)$ \\
Lower limb & $13(44.8)$ & $4(33.3)$ & 0 \\
Fungated lesion & $25(86.2)$ & $2(16.7)$ & 0 \\
Muscle invasion & $12(41.4)$ & $6(50.0)$ & $1(25.0)$ \\
Bone invasion & $9(31.0)$ & & $2(50)$ \\
Regional node & $8(27.6)$ & & \\
Stage & $9(31.0)$ & $4(33.3)$ & $2(50.0)$ \\
I and II & $20(69.0)$ & $8(67.7)$ & $2(50.0)$ \\
III & & & \\
\hline
\end{tabular}

SCC: Squamous cell carcinoma. 
Table 2. Surgical treatment for skin cancer.

\begin{tabular}{cccccc}
\hline Type & SCC & M & Sarcoma & Porocarcinoma & Total \\
\hline Simple excision & 1 & - & 1 & - & 2 \\
Wide excision & 19 & 9 & 2 & 1 & 31 \\
Amputation/disarticulation & 9 & 3 & 1 & - & 13 \\
Lymph node dissection & 9 & 7 & - & - & 16 \\
\hline
\end{tabular}

SCC: Squamous cell carcinoma; M: Melanoma.

were 9 albinos who presented 17 lesions, approximately a total of 1.8 lesions per albino. Male to female ratio was 1:1.4 and median age was 48.0 year old (IQR 28.0 - 60.0). Before admission, 8 (41.4\%) underwent repetitive surgery once (5 cases) and twice ( 3 cases). The tumor was fungated in 25 cases $(86.2 \%)$ and infected in 3 cases (10.3\%). The median tumor size was $7.0 \mathrm{~cm}$ (IQR $3.6-12.0$ ). There was a muscle infiltration in 12 cases (41.4\%) and bone in 9 cases (31.0\%). Inguinal lymph node involvement was found in 8 cases (27.6\%). All regional lymph node involvement were associated with ulcerated lesions. The tumor was classified as stage I and II in 9 cases (31.0\%) and stage III in 20 cases (69.0\%). Skin cancer diagnosis was obtained on biopsy specimen in 23 cases $(50.0 \%)$ and after surgical resection in 23 other cases. The underlying diseases found were: diabetes ( 3 cases), hypertension ( 1 case) and euthyroid goiter ( 1 case).

Surgical treatment included simple excision in 1 case (3.4\%), wide excision in 19 cases $(65.5 \%)$ and amputation or disarticulation in 9 cases $(31.0 \%)$. The inguinal lymph node dissection performed in 9 patients (31.0\%). To close the wound, we used a skin flap in 8 cases and a skin graft in 2 cases. A directed healing was necessary in 2 cases. In 24 other lesions, the closure of the surgical wound was direct. Twenty-one (72.4\%) patients were hospitalized for a duration ranging from 1 to 56 days with a median of 5.0 days (IQR 1.0 - 7.0). Surgical complications occurred in $5(17.4 \%)$ patients, including 3 wound suppurations, 3 lower limb lymphedemas, 1 seroma and 1 skin flap necrosis. Three patients among the patients who had complications had undergone lymph node dissection. Two (6.9\%) patients underwent chemotherapy. Surgical margins were free in 19 (65.5\%) patients, infiltrated in 1 patient (3.4\%) and unspecified in 8 patients (27.5\%). The median follow-up after surgery was 26.0 months (IQR 14.4 - 37.5).

During follow-up, 6 patients $(20.7 \%)$ experienced a relapse. The relapse rate was $10.5 \%$ for free margins, compared with $44.4 \%$ for the other margins status $(p=0.032)$. Twenty patients were alive at median 26 months after surgery, corresponding to $69.0 \%$ of overall survival. The factors associated to survival in univariate analysis were: iterative resection $25.0 \%$ vs $85.7 \%(p=0.004)$, fungated tumor $64.0 \%$ versus $100 \%(p=0.280)$, free surgical margins $84.2 \%$ vs $33.3 \%$ ( $p=$ $0.013)$ and relapse 16.6 versus $82.2 \%(p=0.005)$. In multivariate analysis, the status of surgical margins was the only independent prognostic factor.

\section{Melanoma}

Among 12 melanoma, median age was 71.0 (IQR 40.5 - 76.5) with male to fe- 
male ratio 1:2. Lower limb was primary site in $10(83.4 \%)$. Four (33.3\%) patients underwent repetitive surgery. There were fungated lesions in 9 (75.0\%). Adjacent muscle involved in 4 cases (33.3\%) and bone in 2 cases (16.7\%). Inguinal lymph node involvement was found in 6 cases $(50.0 \%)$. Tumor stages were I and II in 4 (33.3\%) and III in 8 (67.7\%).

Wide excision and amputation or disarticulation performed respectively in 9 (75.0\%) cases and 3 (25.0\%). Seven (58.3\%) patients underwent inguinal lymph node dissection. The wound closure required a direct suture in 4 cases (33.3\%), a directed healing in 7 (58.3\%) cases and a skin flap in $1(8.4 \%)$ case. The median length of stay of the 8 hospitalized patients was 6 days (IQR $1.0-6.0$ ). Complications occurring in 3 patients were 2 wound infection, 1 seroma and 1 lymphedema of lower limb. Two (16.7\%) patients underwent chemotherapy. Surgical margins were free in $6(50.0 \%)$ patients, involved in 2 patients $(16.7 \%)$ and unspecified in 4 patients (33.3\%). During 33 months [IC95\% 1.5 - 64.4] median follow-up after surgery, 6 (50.0\%) experienced relapse and 5 (41.7\%) died. No factor was found associated with risk of relapse. Therefore, all patients with free margins were alive against only $16.7 \%$ with others margins status $-p=0.008$ ). No others factors (relapse, iterative excision) associated with survival.

\section{Sarcoma}

Median age for the 4 sarcoma was 47.5 (IQR 37.5 - 52.2). There was 1 male to 3 females. One of the patients was an albino. All cases were fungated. Tumor stage was I and II in 2 cases and III in 2 others cases. Primary site was head in 2 cases and foot in 2 cases.

These sarcoma treated by simple excision ( 1 case), wide excision ( 2 cases) and amputation (1 case). No lymph node dissection performed. Wound closure obtained by direct suture ( 3 cases) and skin flap (1 case). No complications occurred. Two patients hospitalized for 1 and 7 days. Surgical margins were free in 2 cases and unspecified in 2 cases. Two patients died and 2 were alive after 35 months median follow up.

\section{Porocarcinoma}

The case of porocarcinoma was a 50-year-old woman who presented a skin nodule $2 \mathrm{~cm}$ on the thorax. She underwent wide excision with margin free. She was alive without relapse after an 11-month follow-up.

\section{Follow up for all cases}

The median follow up for all cases was 29.0 month (IQR 59.0 - 7.0). Overall survival was $65.2 \%$ (Figure 1). Factors related to survival in univariate analysis were: iterative resection $(p=0.008)$, fungated tumor $(p=0.037)$, the status of surgical margins $(p=0.002)$ and the occurrence of relapse $(p=0.0000)$. In multivariate analysis, the status of surgical margins was the only independent prognostic factor.

\section{Discussion}

Data showed high frequency of squamous cell carcinomas and melanomas; more 


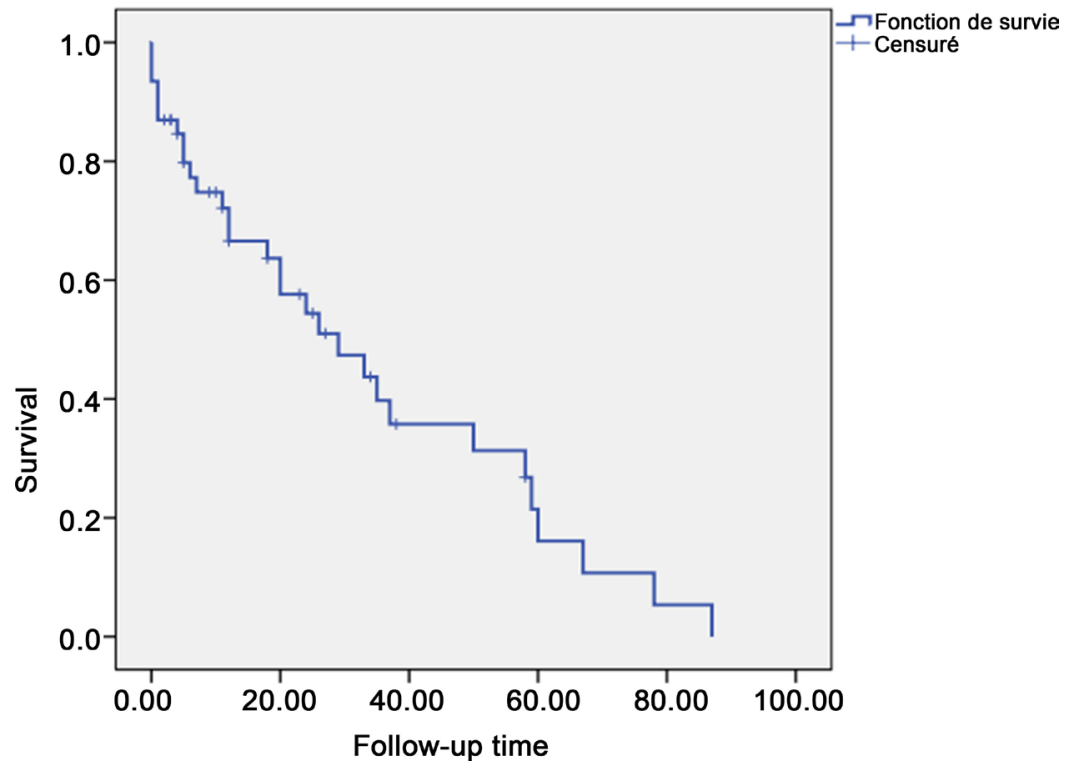

Figure 1. Overall survival for all skin cancers.

than half of the cases in the lower limbs, stage II or beyond. For the non-albinos, there was only one lesion, almost all of which was on the lower limbs. In albinos, the lesions were multiple and localized on the parts exposed sun in albinos; 1.7 lesions per patient in our study, 1.9 according.

The surgical treatment of choice of cutaneous cancers is wide excision. In the case of invasion of adjacent structures and when the function of the limb was compromised, an amputation or disarticulation was then performed. Amputations and disarticulations were both curative and palliative before these tumors ulcerated, sometimes haemorrhagic and very painful. This surgical strategy is reported in several studies [7] [9] [10]. However, because of the reluctance of many of the patients to accept amputation as an option, less adequate local excision was done. This refusal of surgical treatment explains the low number of patients operated in this study. Cases of refusal of amputation were reported by Pisoh et al. [11].

The mainstay of treatment for skin cancers remains surgical excision, with margins determined by histological type and depth of the tumor. Surgical margins of 1 to $2 \mathrm{~cm}$ considered as free for all types of skin cancers in our study. The surgical margins depend on the type of cutaneous cancers: $\geq 1 \mathrm{~cm}$ for SCC, 0.5 $2 \mathrm{~cm}$ for melanoma [7] and $2 \mathrm{~cm}$ or more for sarcoma [12]. Mohs micrographic surgery is an outpatient procedure that maximizes surgical margin evaluation while minimizing the amount of tissue that must be excised [13] [14].

The closure of the operative wound was direct for the majority of cases in this study. In some cases, a skin flap or skin graft has been necessary in order to respect the carcinologic requirements and to repair the aesthetic damage. These artifices concerned the localizations of the head and the neck. The direct closure of the wound may also be compromised by initial non-carcinologic excision.

We performed inguinal lymph node dissection, in addition to the removal of 
the primary tumor. Lymph node dissection has been systematic in the presence of inguinal nodes in 14 cases and the absence of nodes in 2 cases. In the absence of inguinal nodes, we should perform the sentinel lymph node as suggested by many authors [15] [16]. In the presence of the sentinel positive lymph node, lymph node dissection will be performed systematically. In all our cases of lymph node dissection, there was a node involvement. The sentinel lymph node should be a challenge in our practice with the awareness of the early discovery of these cancers. In fact, the sentinel lymph node is able to reduce the morbidity associated with the dissection and to select the patients who must benefit from the lymph node dissection.

The rate of surgical complications of $17.4 \%$ in our study is similar to that of Deo et al which was 15.5\%. These surgical complications occurred in 5 cases of 8 having lymph node dissection in our study.

Immediate surgical complications were wound infections, seroma and cutaneous necrosis. These immediate operative complications were previously reported [7] [17]. In the long term, complications were lymphedema, linked to inguinal lymph node dissection.

In this study, the relapse rate was high, $28.8 \%$ after median 15 months followup; whereas it was $15.0 \%$ in 5 years according to Deo et al. [7]. Despite the amputation performed by Parsons et al. [17], 89\% of patients with healthy surgical margins had recurrences after a median follow-up time of 9.2 months. The median 15 months overall survival (65.2\%) was very low as compared to that of Deo et al [7] who found 75\% survival after 5 years follow up. These differences in survival and relapse could be explained by the advanced stages and the absence of adjuvant radiation therapy in our cases. There was no difference in survival between histologic types whereas others authors [7] found a better survival of squamous cell carcinomas than melanomas. The survival of cutaneous melanoma of the lower limbs was 35\% in Nigeria [9]. Surgical margins were the only independent prognostic factor. These findings suggest that poor initial surgery may compromise locoregional control of disease.

\section{Conclusion}

This study showed that the prognosis of skin cancers depends on the quality of the initial excision. Early diagnosis can prevent the advanced stages.

\section{Acknowledgements}

None to disclose.

\section{Funding}

None to disclose.

\section{Competing Interests}

The authors declare that they have no competing interests. 


\section{Declarations}

None to disclose.

\section{Availability of Data and Materials}

The datasets during and/or that were analyzed during the current study are available from the corresponding author upon reasonable request.

\section{Ethics Approval and Consent to Participate}

In this retrospective study, data were collected anonymously and confidentially. Patients signed the consent form for the use of data contained in their records.

\section{Consent for Publication}

Not applicable.

\section{References}

[1] Traore, B., Keita, M., Conde, M., et al. (2016) Anatomo-Clinical Characteristics of Skin Cancers at the Surgical Oncology Unit of the Conakry University Hospital. Rev. CAMES SANTE, 4, 79-82

[2] Clairwood, M., Ricketts, J., Grant-Kels, J. and Gonsalves, L. (2014) Melanoma in Skin of Color in Connecticut: An Analysis of Melanoma Incidence and Stage at Diagnosis in Non-Hispanic Blacks, Non-Hispanic Whites, and Hispanics. International Journal of Dermatology, 53, 425-33.

[3] Eisemann, N., Waldmann, A., Geller, A.C., et al. (2014) Non-Melanoma Skin Cancer Incidence and Impact of Skin Cancer Screening on Incidence. Journal of Investigative Dermatology, 134, 43-50.

[4] Asuquo, M.E. and Ebughe, G. (2012) Major Dermatological Malignancies Encountered in the University of Calabar Teaching Hospital, Calabar, Southern Nigeria. International Journal of Dermatology, 51, 36-40.

[5] Forae, G.D. and Olu-Eddo, A.N. (2013) Malignant Skin Tumors in Benin City, South-South, Nigeria. Oman Medical Journal, 28, 311-315.

[6] Oseni, G.O., Olaitan, P.B., Komolafe, A.O., et al. (2015) Malignant Skin Lesions in Oshogbo, Nigeria. Pan African Medical Journal, 20, 253.

[7] Deo, S.V., Hazarika, S., Shukla, N.K., et al. (2005) Surgical Management of Skin Cancers: Experience from a Regional Cancer Centre on North India. Indian Journal of Cancer, 42, 145-150.

[8] Sobin, L.H., Gospodarowicz, M.K. and Wittekind, C. International Union against Cancer. TNM Classification of Malignant Tumours. 7th ed, 2009, West Sussex, Chichester, UK, 2010, Wiley-Blackwell, Hoboken, NJ.

[9] Gualdi, G., Monari, P., Apalla, Z. and Lallas, A. (2015) Surgical Treatment of Basal Cell Carcinoma and Squamous Cell Carcinoma. Giornale Italiano Di Dermatologia E Venereologia, 150, 435-447.

[10] Parsons, C.M., Pimiento, J.M., Cheong, D., et al. (2012) The Role of Radical Amputations for Extremity Tumors: A Single Institution Experience and Review of the Literature. Journal of Surgical Oncology, 105, 149-155. https://doi.org/10.1002/jso.22067 
[11] Pisoh, T.C., Ngowe Ngowe, M., Watcoun-Nchinda, E.P., et al. (2011) Surgical Treatment of Lower Limb Malignant Melanoma IN Yaounde Cameroon. Health Sciences and Disease, 12.

[12] Kandel, R., Coakley, N., Werier, J., et al. (2013) Surgical Margins and Handling of Soft-Tissue Sarcoma in Extremities: A Clinical Practice Guideline. Current Oncology, 20, e247-e254. https://doi.org/10.3747/co.20.1308

[13] Lane, J.E. and Kent, D.E. (2005) Surgical Margins in the Treatment of Nonmelanoma Skin Cancer and Mohs Micrographic Surgery. Journal of Surgical Education, 62, 518-526. https://doi.org/10.1016/j.cursur.2005.01.003

[14] Ad Hoc Task Force, Connolly, S.M., Baker, D.R., Coldiron, B.M. and AAD/ACMS/ASDSA/ASMS (2012) Appropriate Use Criteria for Mohs Micrographic Surgery: A Report of the American Academy of Dermatology, American College of Mohs Surgery, American Society for Dermatologic Surgery Association, and the American Society for Mohs Surgery. Journal of the American Academy of Dermatology, 67, 531-550.

[15] Ul-Mulk, J. and Hölmich, L.R. (2012) Lymph Node Dissection in Patients with Malignant Melanoma Is Associated with High Risk of Morbidity. Danish Medical Journal, 59, A4441.

[16] Matthey-Giè, M.-L., Boubaker, A., Letovanec, I., et al. (2013) Sentinel Lymph Node Biopsy in Nonmelanoma Skin Cancer Patients. Journal of Skin Cancer, 2013, Article ID: 267474.

[17] Opara, K.O. and Jiburum, B.C. (2010) Skin Cancers in Albinos in a Teaching Hospital in Eastern Nigeria - Presentation and Challenges of Care. World Journal of Surgical Oncology, 8, 73.

\section{Abbreviations}

IQR: Interquartile range

SCC: Squamous Cell Carcinoma

TNM: Tumor Node Metastasis

UICC: International Union for the Control of Cancer 